\title{
A focused simulation-based optimization of print time and material usage with respect to orientation, layer height and support settings for multi-pathological anatomical models in inverted vat photopolymerization 3D printing
}

\author{
Prashanth Ravi ${ }^{1 *}$ id and Victoria C. P. Chen ${ }^{2}$
}

\begin{abstract}
Background: 3D printing of anatomical models requires multi-factorial decision making for optimal model manufacturing. Due to the complex nature of the printing process, there are frequently multiple potentialities based on the desired end goal. The task of identifying the most optimal combination of print control variables is inherently subjective and rests on sound operator intuition. This study investigates the effect of orientation, layer and support settings on print time and material usage. This study also presents a quantitative optimization framework to jointly optimize print time and material usage as a function of those settings for multi-pathological anatomical models.

Methods: Seven anatomical models representing different anatomical regions (cardiovascular, abdominal, neurological and maxillofacial) were selected for this study. A reference cube was also included in the simulations. Using PreForm print preparation software the print time and material usage was simulated for each model across 4 orientations, 2 layer heights, 2 support densities and 2 support tip sizes. A 90-10 weighted optimization was performed to identify the 5 most optimal treatment combinations that resulted in the lowest print time (90\% weight) and material usage (10\% weight) for each model.

Results: The $0.1 \mathrm{~mm}$ layer height was uniformly the most optimal setting across all models. Layer height had the largest effect on print time. Orientation had a complex effect on both print time and material usage in certain models. The support density and the support tip size settings were found to have a relatively minor effect on both print time and material usage. Hollow models had a larger support volume fraction compared to solid models.
\end{abstract}

* Correspondence: prashanth.ravi@uc.edu

${ }^{1}$ Department of Radiology, University of Cincinnati College of Medicine, 234 Goodman St, Cincinnati, OH 45219, USA

Full list of author information is available at the end of the article

(c) The Author(s). 2021, corrected publication 2021. Open Access This article is licensed under a Creative Commons Attribution 4.0 International License, which permits use, sharing, adaptation, distribution and reproduction in any medium or format, as long as you give appropriate credit to the original author(s) and the source, provide a link to the Creative Commons licence, and indicate if changes were made. The images or other third party material in this article are included in the article's Creative Commons licence, unless indicated otherwise in a credit line to the material. If material is not included in the article's Creative Commons licence and your intended use is not permitted by statutory regulation or exceeds the permitted use, you will need to obtain permission directly from the copyright holder. To view a copy of this licence, visit http://creativecommons.org/ licenses/by/4.0/. The Creative Commons Public Domain Dedication waiver (http://creativecommons.org/publicdomain/zero/1. 0/) applies to the data made available in this article, unless otherwise stated in a credit line to the data. 
Conclusions: The quantitative optimization framework identified the 5 most optimal treatment combinations for each model using a 90-10 weighting for print time and material usage. The presented optimization framework could be adapted based on the individual circumstance of each 3D printing lab and/or to potentially incorporate additional response variables of interest.

Keywords: Model orientation, Layer height, Medical 3D Printing, Print time, Material usage, Optimization, Inverted vat photopolymerization, Anatomical Models

\section{Background}

Three-dimensional printing is increasingly employed to manufacture patient-specific anatomical models, medical devices and simulation models for training $[1,2]$. There are several categories of $3 \mathrm{D}$ printing technology. The inverted Vat Photopolymerization (VP) technology is being used more frequently within academic medical centers due to its relative affordability as hospitals invest in point-of-care 3D printing facilities. There are two main types of VP technology including top-down laser-based VP generally used in industrial machines with large build volumes, and bottom-up laser based VP with relatively smaller machines also frequently referred to by the terminology desktop stereolithography (SLA) [3]. Bottomup LCD/DLP VP printers are also available in the desktop segment. The technology employed in this paper is the bottom-up laser-based VP used in the Formlabs 3D printers [4]. Given that 3D printing is a complex multifactorial process, there are often trade-offs between desired end goals. For instance, model accuracy and surface quality can be improved by reducing the layer height and/or re-orienting the model, but frequently at the expense of increased print time and cost [5-7]. Furthermore, the same control variables used across models can yield different output responses highlighting the model-specific nature of the manufacturing process [8]. The organic and complex model geometries present in anatomical models offer further unique challenges in such decision making. Advanced technologies such as machine learning and novel support generation strategies have been employed in 3D printing to optimize response variables such as energy consumption and material waste [9-11].

Kamio et al. found that an increase in layer height reduced the print time and material cost for a mandibular model without significant reduction in geometric accuracy [12]. Rubayo et al. found a significant difference in print time between dental surgical templates manufactured vertically vs. horizontally [13]. Some research groups have studied the accuracy of medical models and found the accuracy to be affected by multiple factors such as orientation, layer height and the cure settings [4, 14-17]. However, a focused investigation of the effect of different model geometries and critical print variables on important response variables is lacking in the medical
3D printing literature. Furthermore, the decision-making scheme surrounding model orientation and choice of print settings to achieve desired end goals remains unclear for the 3D printing of anatomical models and implants. For instance, optimizing for print time is crucial when a $3 \mathrm{D}$ printed model is needed for an emergency or trauma case.

The goal of this research was to investigate the effect of model orientation, layer height, support density and support tip size on print time and material usage in VP 3D printing based on print simulation for 7 anatomical models. We propose an adaptable optimization framework to jointly optimize both print time and material usage which could be tailored based on the availability of resources at the 3D printing laboratory. The framework enables the identification of optimal combinations of control variables using a quantitative approach and could serve as a useful guide to the 3D printer operator. The optimization scheme could also be further extended to include other response variables of interest based on the desired end goal.

\section{Methods}

\section{Image segmentation and model STL creation}

Image sets across anatomical regions and pathologies were selected for this study as part of the clinical 3D printing service line at the University of Cincinnati Department of Radiology. These included models for: left atrial appendage (LAA) occluder device sizing, minimally invasive coronary artery bypass (MICAB) surgery, low grade glioma (LGG) excision, renal cell carcinoma (RC) surgery, mandibular osteonecrosis (MO) resection, hepatic pseudoaneurysm (HPA) surgery and basilar tip aneurysm (BTA) clipping (Fig. 1). The models represent a range of volumes from $\sim 2 \mathrm{cc}$ to $\sim 100 \mathrm{cc}$ (Table 1). All data was completely de-identified and the research was submitted to the hospital research ethics board, and the study was considered exempt from further review, based on the fact that no data in this study could be used to identify any human being. The relevant anatomical regions were segmented from CT and MRI derived DICOM images using Materialise Mimics InPrint 3.0 (Materialise, Leuven, Belgium). The thresholding presets for Bone (226 to $3071 \mathrm{HU}$ ), Blood Vessel (200 to 3071 HU) and Kidneys (20 to $135 \mathrm{HU}$ ) within the software 


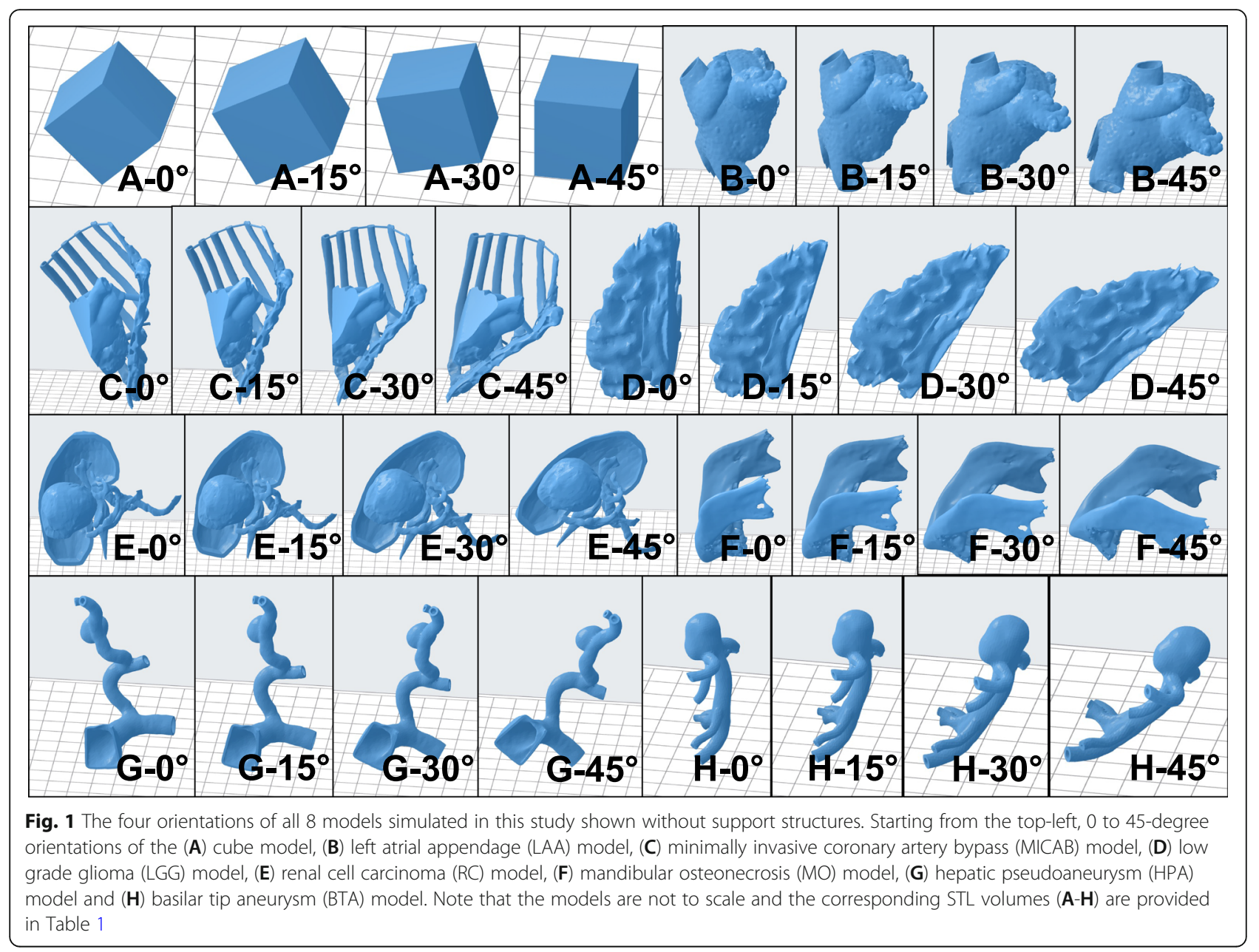

were used for CT images. The segmentation of relevant anatomical regions from MRI were performed using semi-automatic and manual segmentation methods. The LAA, HPA and BTA models were generated by constructing a $1.00-1.50 \mathrm{~mm}$ wall around the segmented blood pool. All other models including the reference cube were fully solid. The STL files were post-processed

Table 1 The 8 models investigated in this study with their corresponding STL volumes and internal geometry type

\begin{tabular}{llll}
\hline Label & Model & Type & $\begin{array}{l}\text { STL Volume } \\
\text { (cc) }\end{array}$ \\
\hline A & Cube (reference) & Solid & 8.00 \\
B & Left atrial appendage (LAA) & Hollow & 30.25 \\
C & $\begin{array}{l}\text { Minimally invasive coronary artery } \\
\text { bypass (MICAB) }\end{array}$ & Solid & 100.33 \\
D & Low grade glioma (LGG) & Solid & 86.14 \\
E & Renal cell carcinoma (RC) & Solid & 73.92 \\
F & Mandibular osteonecrosis (MO) & Solid & 67.55 \\
G & Hepatic pseudoaneurysm (HPA) & Hollow & 3.47 \\
H & Basilar tip aneurysm (BTA) & Hollow & 1.98 \\
\hline
\end{tabular}

in Materialise 3-matic 15.0 (Materialise, Leuven, Belgium) where they were fixed, smoothed and wrapped. The smoothing factor was set to 0.90 . Spike removal was performed on the $\mathrm{RC}$ and MO models with the spike size and smallest detail both set to $0.25 \mathrm{~mm}$. The LAA model consisted of the appendage, atrium and pulmonary veins; the MICAB model consisted of the heart, left anterior descending artery, left interior mammary artery,

Table 2 Summary of the Print Time (PT), Material Usage (MU) and average support to total volume ratio for all 8 models

\begin{tabular}{llll}
\hline Model & $\begin{array}{l}\text { Avg. PT } \pm \text { Std. } \\
\text { Dev. }(\text { min) }\end{array}$ & $\begin{array}{l}\text { Avg. } \mathbf{M U} \pm \text { Std. } \\
\text { Dev. }(\mathrm{mL})\end{array}$ & $\begin{array}{l}\text { Avg. Support/Total } \\
\text { Volume (\%) }\end{array}$ \\
\hline Cube & $109.8 \pm 33.3$ & $10.3 \pm 0.4$ & 22.3 \\
LAA & $680 \pm 188.6$ & $56.5 \pm 3.5$ & 46.5 \\
MICAB & $876.1 \pm 252.5$ & $129.5 \pm 3.3$ & 22.5 \\
LGG & $565.3 \pm 156.8$ & $100.7 \pm 5.0$ & 14.5 \\
RC & $678.1 \pm 198.0$ & $94.4 \pm 1.8$ & 21.7 \\
MO & $610.2 \pm 169.8$ & $87.8 \pm 2.6$ & 23.1 \\
HPA & $272.7 \pm 98.2$ & $7.3 \pm 0.8$ & 52.5 \\
BTA & $165.9 \pm 52.5$ & $5 \pm 0.4$ & 60.4 \\
\hline
\end{tabular}




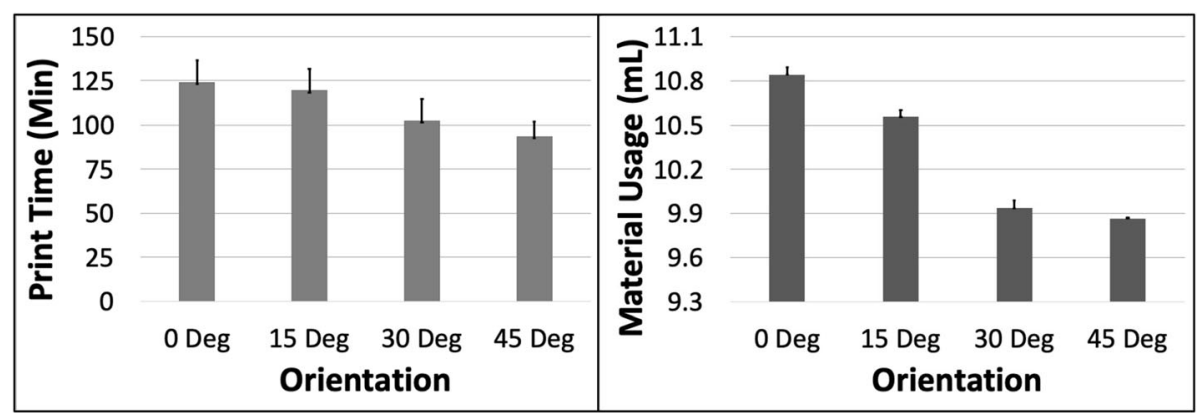

Fig. 2 The response variables Print Time (PT) and Material Usage (MU) plotted against Orientation (ORN) for the Cube model. Sample size is 8 samples for each PT and MU average and the associated error bar

sternum and ribs; the LGG model consisted of the glioma and white matter; the RC model consisted of the tumor, kidney shell and arterial as well as venous branches; the MO model consisted of the lower mandible; the HPA and BTA models consisted of the vessel wall and corresponding aneurysms (Fig. 1).

\section{Study design and print simulation in PreForm}

The study consisted of 8 models spanning a range of volumes, anatomical regions/pathologies and included 3 hollow models (Table 1). A $20 \mathrm{~mm} \times 20 \mathrm{~mm} \times 20 \mathrm{~mm}$ cube was included as reference geometry. The Print Time (PT) and Material Usage (MU) for each model was simulated in PreForm 3.9.0 (Formlabs, Somerville, MA, USA) with the resin set to Clear and printer set to the Form 3 (Formlabs, Somerville, MA, USA). Clear resin was used since it is the material of choice for most anatomical models at our lab because it allows the visualization of internal features. The PT and MU values included the model, support structure as well as raft for each print simulation. Four model orientations (ORN) $0,15,30,45$ degrees, two layer heights $(\mathrm{LH})-0.05,0.10$ $\mathrm{mm}$, two support densities (SD) $-0.80,1.00$ and two support tip sizes (STS) $-0.4,0.5 \mathrm{~mm}$ were tested for a total of 32 treatment combinations $(4 \mathrm{ORN} \times 2 \mathrm{LH} \times 2 \mathrm{SD} \times 2 \mathrm{STS})$ per model. The 4 orientation levels were chosen to represent common model orientation choices in inverted VP 3D printing. The 2 layer heights are most commonly used for 3D printing anatomical models. The lowest $0.025 \mathrm{~mm}$ layer height is typically only used when the highest accuracy is needed such as for surgical guides or implants that form part of an assembly. The 2 support densities and 2 support tip sizes represent the commonly used levels that satisfy "printability" in PreForm while resulting in good surface quality (larger tip sizes result in reduced surface quality). Each of the 32 treatments per model was simulated only once since there is no expected random error associated with this type of simulation. The 0-degree orientation was defined for each model as the most vertical orientation. The models were then rotated about the $\mathrm{X}$-axis (which runs front-to-back in PreForm) in 15-degree increments (Fig. 1). The Z-height therefore reduced with increasing ORN for each model. Each model was placed in the center of the build plate for consistency between simulations. Support structures were generated both inside and outside the model since during actual 3D printing these internal supports would be needed for hollow models.

\section{Data analysis and optimization}

The PT and MU were averaged by the 4 control variables - ORN, LH, SD and STS to assess their effect on

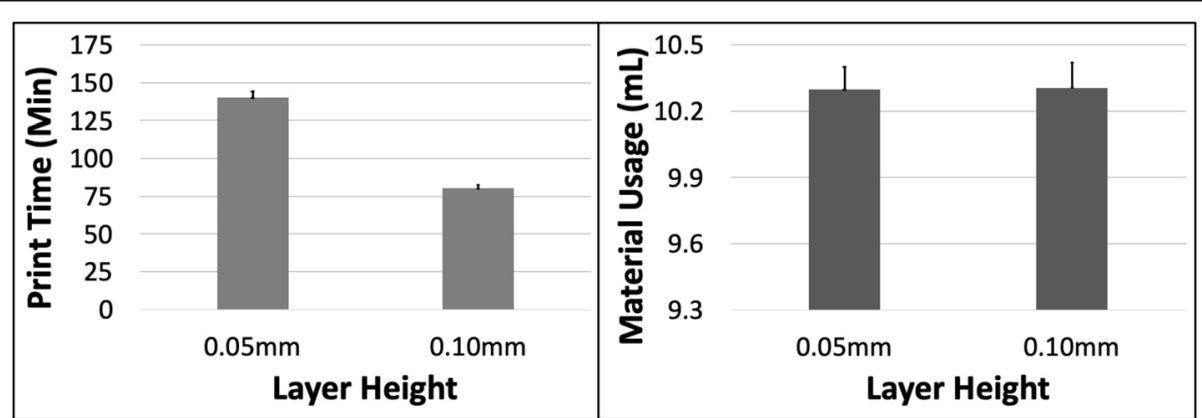

Fig. 3 The response variables Print Time (PT) and Material Usage (MU) plotted against Layer Height (LH) for the Cube model. Sample size is 16 samples for each PT and MU average and the associated error bar 
Table 3 The 5 most weighted (W) optimal treatment combinations for the Cube model

\begin{tabular}{lllllll}
\hline ORN (Deg) & LH $(\mathbf{m m})$ & SD & STS $(\mathbf{m m})$ & MU $(\mathbf{m L})$ & PT $(\mathbf{m i n})$ & W \\
\hline 30 & 0.1 & 0.8 & 0.5 & 9.79 & 67 & -1.0000 \\
30 & 0.1 & 1 & 0.4 & 9.92 & 68 & -0.9586 \\
30 & 0.1 & 1 & 0.5 & 9.97 & 69 & -0.9304 \\
45 & 0.1 & 0.8 & 0.4 & 9.87 & 71 & -0.9075 \\
45 & 0.1 & 1 & 0.4 & 9.87 & 71 & -0.9075 \\
\hline
\end{tabular}

PT and MU. A weighted optimization of PT and MU was performed with a weightage of $90 \%$ to PT and $10 \%$ to MU with the goal to identify the combination of control variables that resulted in the minimal weight. PT was given $90 \%$ weight since it is a more important par-

$$
P T_{\text {Center }}=\frac{\operatorname{Max}(P T)+\operatorname{Min}(P T)}{2}
$$

$P T_{\text {Center }}$ is an intermediate variable to store the average of the endpoints of the PT dataset for a model.

$$
P T_{\text {Bottom }}=\frac{\operatorname{Max}(P T)-\operatorname{Min}(P T)}{2}
$$

$P T_{\text {Bottom }}$ is an intermediate variable to store half the difference between endpoints of the PT dataset for a model.

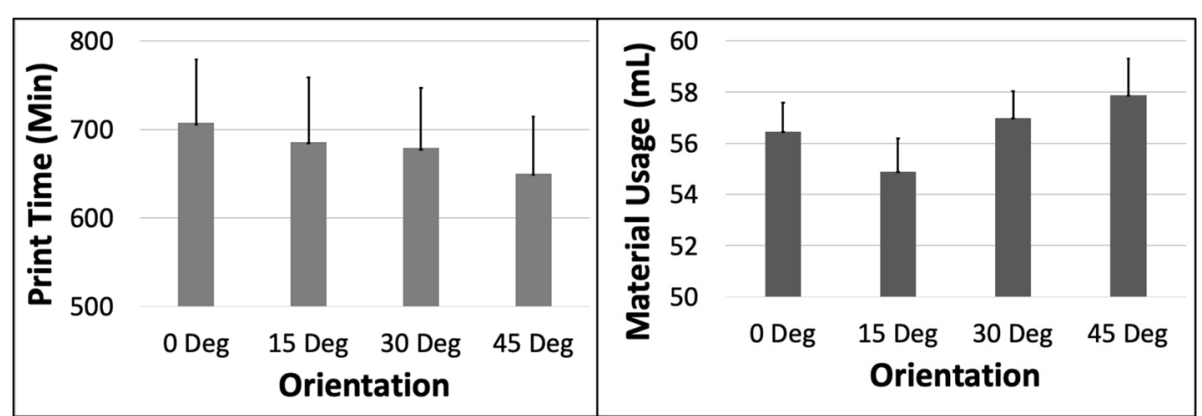

Fig. 4 The response variables Print Time (PT) and Material Usage (MU) plotted against Orientation (ORN) for the LAA model. Sample size is 8 samples for each PT and MU average and the associated error bar

ameter, particularly when 3D printing models for urgent procedures and for smaller $3 \mathrm{D}$ printing laboratories seeking to maximize their throughput. The raw optimization equations below were fed into Microsoft Excel (Microsoft, Redmond, WA, USA) for computation. The 5 most optimal solutions for each of the 8 models were further analyzed. The optimization used the following approach:

$$
P T_{\text {Normalized }}=\frac{P T-P T_{\text {Center }}}{P T_{\text {Bottom }}}
$$

$P T_{\text {Normalized }}$ is an intermediate variable to store the normalized PT of a single PT data point for a model. $M U_{\text {Normalized }}$ was calculated similarly using Eq. 1, Eq. 2 and Eq. 3 but using the corresponding MU values.

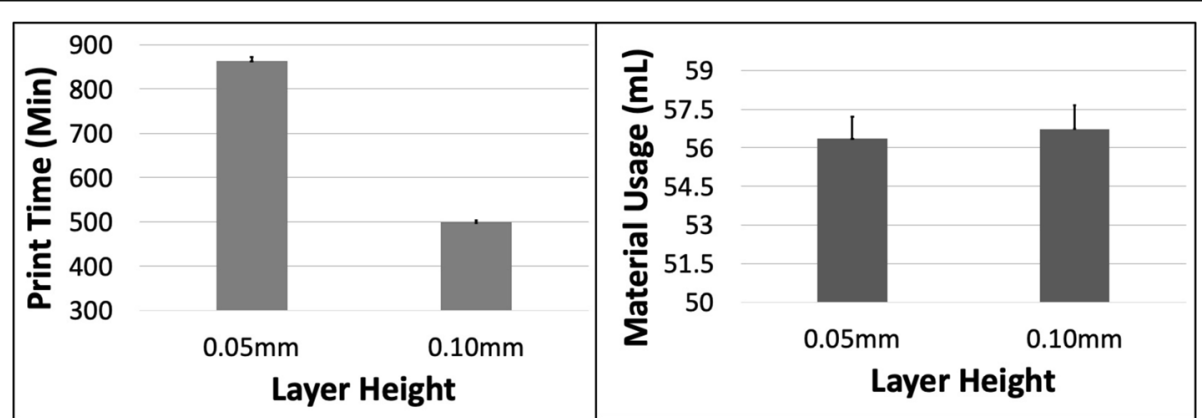

Fig. 5 The response variables Print Time (PT) and Material Usage (MU) plotted against Layer Height (LH) for the LAA model. Sample size is 16 samples for each PT and MU average and the associated error bar 


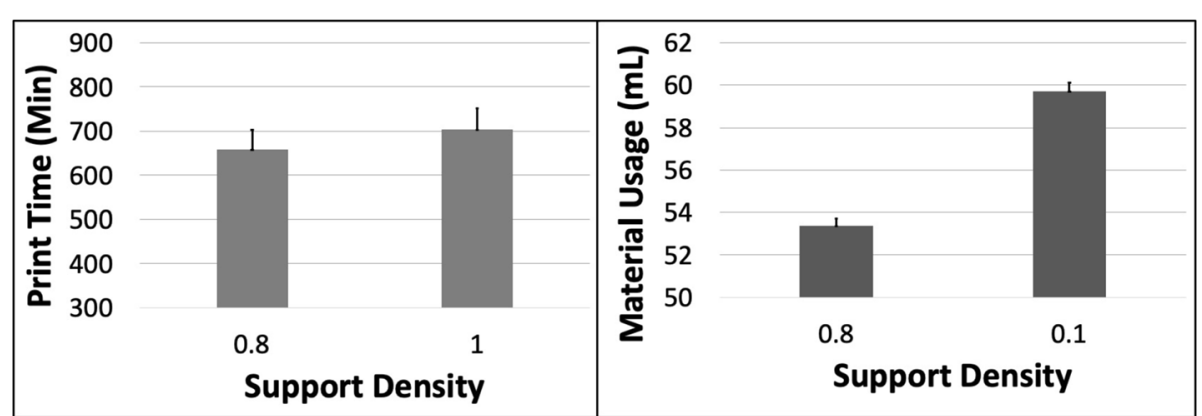

Fig. 6 The response variables Print Time (PT) and Material Usage (MU) plotted against Support Density (SD) for the LAA model. Sample size is 16 samples for each PT and MU average and the associated error bar

$$
W=0.90 \times P T_{\text {Normalized }}+0.10 \times M U_{\text {Normalized }}
$$

W represents the weight of the final optimization. The lower the $\mathrm{W}$, the more optimal the combination of input variables which yield the corresponding PT and MU.

\section{Results}

The average PT ranged from $\sim 2$ to $\sim 14.5 \mathrm{~h}$ and the average MU ranged from $\sim 5$ to $\sim 130 \mathrm{~mL}$ for the 8 models (Table 2). The average support volume as a frac-

Table 4 The 5 most weighted (W) optimal treatment combinations for the LAA model

\begin{tabular}{lllllll}
\hline ORN (Deg) & LH $(\mathbf{m m})$ & SD & STS $(\mathbf{m m})$ & $\mathbf{M U}(\mathbf{m L})$ & PT $(\mathbf{m i n})$ & W \\
\hline 45 & 0.1 & 0.8 & 0.5 & 53.21 & 454 & -0.9639 \\
45 & 0.1 & 0.8 & 0.4 & 54.05 & 456 & -0.9409 \\
15 & 0.1 & 0.8 & 0.5 & 51.24 & 472 & -0.9314 \\
15 & 0.1 & 0.8 & 0.4 & 51.35 & 476 & -0.9141 \\
30 & 0.1 & 0.8 & 0.5 & 54.47 & 480 & -0.8417 \\
\hline
\end{tabular}

tion of total volume ranged from $\sim 15$ to $\sim 60 \%$. This fraction ranged from $\sim 15-23 \%$ for Solid models and $47-60 \%$ for Hollow models. The range of coefficient of variation (std. dev./avg.) was higher for PT (0.28-0.32) than MU (0.02-0.11) across the 8 models.

\section{A - cube}

The PT and MU both reduced with increasing ORN for the Cube model (Fig. 2). The reduction in PT was more substantial $(\sim 25 \%)$ compared to MU $(\sim 10 \%)$ between the 0 and 45 deg levels. An increase in LH had the most impact on PT ( $\sim 43 \%$ reduction) but very little effect on the MU (<0.1\%) (Fig. 3). The 5 most optimal (weighted) treatment combinations for the Cube model all consisted of the 30 and $45 \mathrm{deg}$ ORNs and $0.1 \mathrm{~mm} \mathrm{LH}$ (Table 3). These optimal combinations consisted of both SD and STS levels due to their relatively minor effect on both PT and MU.

\section{B - left atrial appendage (LAA)}

The PT reduced with increasing ORN for the LAA model but the pattern was more complex for MU with an initial reduction followed by a subsequent rise (Fig. 4). The reduction in PT was more substantial $(\sim 8 \%)$ between the 0 and 45 deg levels compared to the increase in MU ( 5\%) between the $15 \mathrm{deg}$ and 45 deg levels. An increase in LH caused a 42\% reduction in PT but barely any change $(<1 \%)$ in $\mathrm{MU}$ (Fig. 5). An increase in SD caused an increase in both PT of $\sim 7 \%$ and in MU of $\sim 12 \%$ (Fig. 6). The 5 most optimal (weighted) treatment combinations consist of the $0.1 \mathrm{~mm} \mathrm{LH}$ and $0.80 \mathrm{SD}$ with mostly larger ORNs

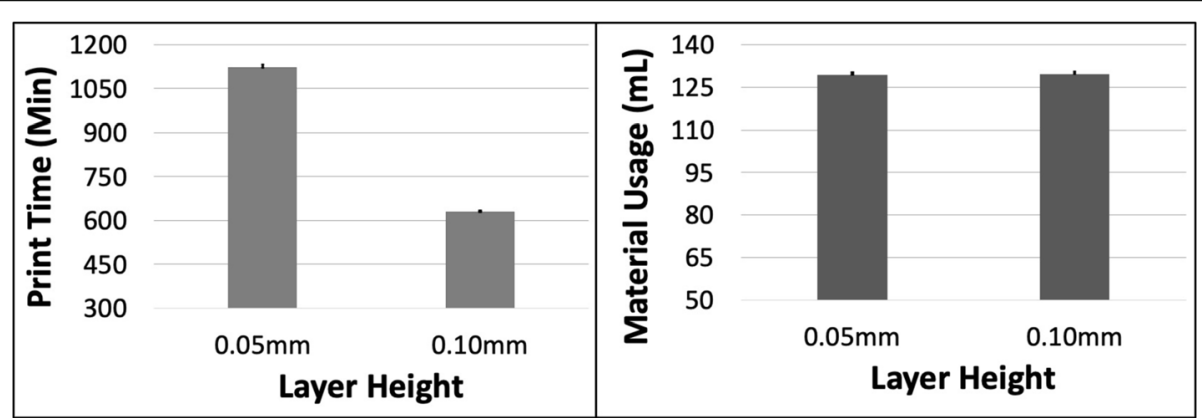

Fig. 7 The response variables Print Time (PT) and Material Usage (MU) plotted against Layer Height (LH) for the MICAB model. Sample size is 16 samples for each PT and MU average and the associated error bar 


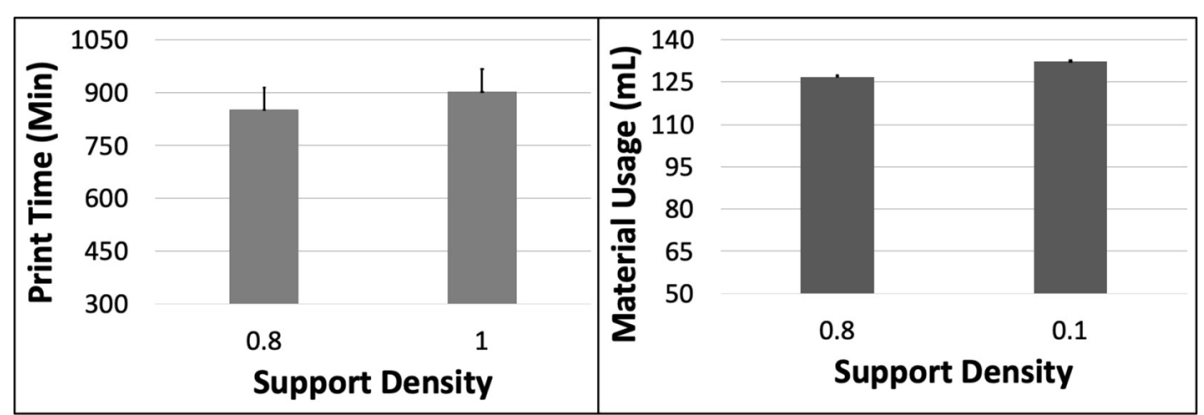

Fig. 8 The response variables Print Time (PT) and Material Usage (MU) plotted against Support Density (SD) for the MICAB model. Sample size is 16 samples for each PT and MU average and the associated error bar

(Table 4), and both levels of STS due to the minor effect of this control variable.

\section{C - minimally invasive coronary artery bypass (MICAB)}

The PT and MU showed little change with ORN (< 3\%) for the MICAB model. The PT reduced by

Table 5 The 5 most weighted (W) optimal treatment combinations for the MICAB model

\begin{tabular}{lllllll}
\hline ORN (Deg) & LH $(\mathbf{m m})$ & SD & STS $(\mathbf{m m})$ & MU $(\mathbf{m L})$ & PT $(\mathbf{m i n})$ & W \\
\hline 15 & 0.1 & 0.8 & 0.5 & 125.6 & 612 & -0.9320 \\
0 & 0.1 & 0.8 & 0.4 & 126.88 & 606 & -0.9309 \\
0 & 0.1 & 0.8 & 0.5 & 126.43 & 611 & -0.9224 \\
30 & 0.1 & 0.8 & 0.5 & 126.34 & 612 & -0.9207 \\
30 & 0.1 & 0.8 & 0.4 & 127.03 & 609 & -0.9194 \\
\hline
\end{tabular}

44\% with increased LH while the MU remained unchanged (Fig. 7). The PT and MU both increased with increasing SD, by $\sim 4.2 \%$ and $5.9 \%$, respectively (Fig. 8). The 5 most optimal (weighted) treatment combinations consist of the $0.1 \mathrm{~mm} \mathrm{LH}$ and $0.80 \mathrm{SD}$ with mostly smaller and larger ORNs (Table 5) as well as both levels of STS due to the minor effect of the latter 2 control variables.

\section{D - low grade glioma (LGG)}

PT and MU both increased with ORN for the LGG model (Fig. 9). The maximum increase in PT was $~ 9.8 \%$ (between 0 and $30 \mathrm{deg}$ ) and in MU was $~ 12.9 \%$ (between 0 and $45 \mathrm{deg})$. The PT reduced by $\sim 42.5 \%$ with increased $\mathrm{LH}$ but the MU remained unchanged (Fig. 10). An increase in SD had a minor impact on both PT and MU (< $2.5 \%$ increase). The 5 most optimal (weighted) treatment combinations consist of the $0.1 \mathrm{~mm} \mathrm{LH}$ and smaller ORNs (Table 6) as well as both levels of STS and SD due to the minor effect of the latter 2 control variables.

\section{E - renal cell carcinoma (RC)}

The PT and MU both reduced with increasing ORN for the RC model (Fig. 11). The maximum reduction was $7.7 \%$ in PT (between 0 and $30 \mathrm{deg}$ ) and $\sim 2.9 \%$ in $\mathrm{MU}$ (between 0 and $30 \mathrm{deg}$ ). The PT reduced by $\sim 44.4 \%$ with increasing $\mathrm{LH}$ while the MU remained constant (Fig. 12). An increase in SD resulted in a $\sim 2 \%$ increase in both PT and MU. An increase in STS resulted in a $1-1.5 \%$ reduction in both $\mathrm{PT}$ and MU. The 5 most

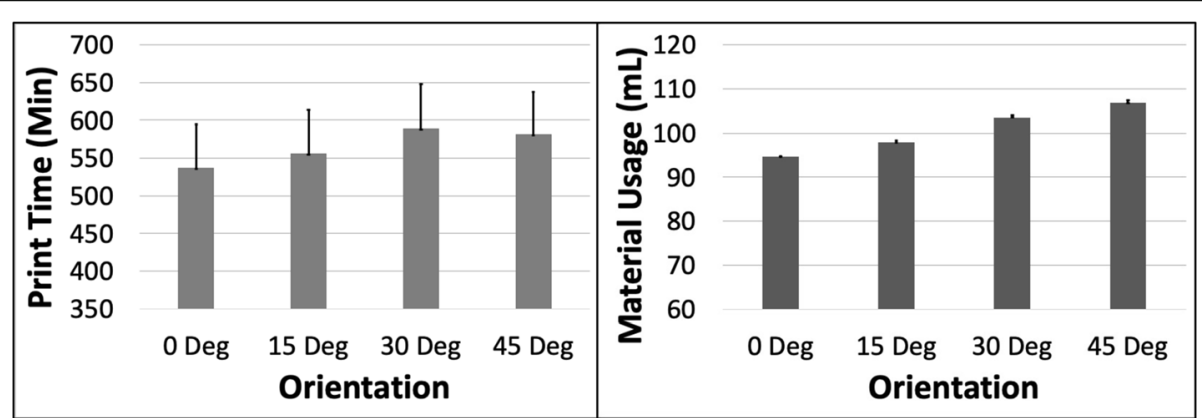

Fig. 9 The response variables Print Time (PT) and Material Usage (MU) plotted against Orientation (ORN) for the LGG model. Sample size is 8 samples for each PT and MU average and the associated error bar 


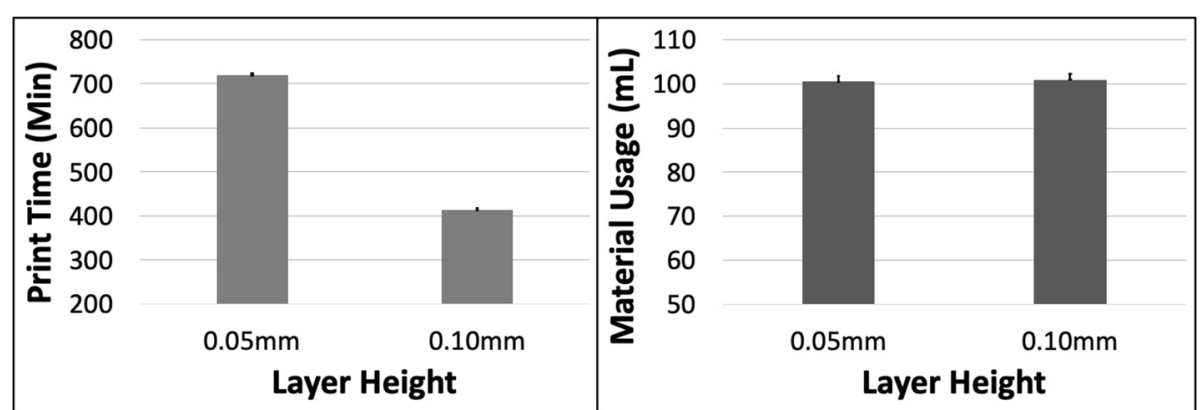

Fig. 10 The response variables Print Time (PT) and Material Usage (MU) plotted against Layer Height (LH) for the LGG model. Sample size is 16 samples for each PT and MU average and the associated error bar

optimal (weighted) treatment combinations consist of the $0.1 \mathrm{~mm} \mathrm{LH}$ and larger ORNs (Table 7) as well as both levels of STS and SD due to the relatively minor effect of the latter 2 control variables.

\section{F - mandibular osteonecrosis (MO)}

The PT reduced and MU increased with increasing ORN (Fig. 13). The maximum reduction in PT was 4.6\% (between 0 and $45 \mathrm{deg}$ ) and the maximum increase in $\mathrm{MU}$ was $\sim 6.2 \%$ (between 0 and $45 \mathrm{deg}$ ) for the $\mathrm{MO}$ model. An increase in LH resulted in a reduction in PT of $\sim 42.8 \%$ with constant MU. The PT increased $\sim 3.5 \%$ and MU increased $\sim 3.2 \%$ when the SD was increased (Fig. 14). The 5 most optimal (weighted) treatment combinations consist of the $0.1 \mathrm{~mm} \mathrm{LH}, 0.8 \mathrm{SD}$ and smaller ORNs (Table 8) with both levels of STS due to the relatively minor effect of the latter control variable.

\section{G - hepatic pseudoaneurysm (HPA)}

The PT and MU both showed a complex pattern with respect to ORN (Fig. 15). The PT first reduced $\sim 5.4 \%$ from 0 to 15 Deg ORN and subsequently increased by 11.6\% from 15 to 45 Deg ORN. The MU likewise reduced $\sim 15.8 \%$ from 0 to 15 Deg ORN and later increased by $\sim 22.7 \%$ from 15 to 45 Deg ORN. The PT reduced by $\sim 52 \%$ with increased $\mathrm{LH}$ while $\mathrm{MU}$ reduced by $\sim 2.5 \%$. The MU increased $\sim 13 \%$ with increased SD whereas PT increased $\sim 1.2 \%$ (Fig. 16). The 5 most optimal (weighted) treatment combinations consist of the $0.1 \mathrm{~mm} \mathrm{LH}, 0.8 \mathrm{SD}$ and both smaller and larger ORNs

Table 6 The 5 most weighted (W) optimal treatment combinations for the LGG model

\begin{tabular}{lllllll}
\hline ORN (Deg) & LH (mm) & SD & STS $(\mathbf{m m})$ & MU $(\mathbf{m L})$ & PT (min) & W \\
\hline 0 & 0.1 & 0.8 & 0.5 & 93.75 & 378 & -1.0000 \\
0 & 0.1 & 0.8 & 0.4 & 94.2 & 381 & -0.9799 \\
0 & 0.1 & 1 & 0.5 & 94.58 & 384 & -0.9607 \\
0 & 0.1 & 1 & 0.4 & 95.13 & 389 & -0.9297 \\
15 & 0.1 & 0.8 & 0.4 & 97.21 & 399 & -0.8550 \\
\hline
\end{tabular}

(Table 9) with both levels of STS due to the relatively minor effect of the latter control variable.

\section{$\mathrm{H}$ - basilar tip aneurysm (BTA)}

The PT reduced and MU increased with ORN (Fig. 17). The PT reduced by $\sim 11.3 \%$ and $\mathrm{MU}$ increased by $20.5 \%$ from 0 Deg to 45 Deg ORN. The PT reduced by $\sim 47 \%$ with increased $\mathrm{LH}$ while $\mathrm{MU}$ increased by $\sim 3.8 \%$. The MU increased $\sim 5.3 \%$ with increased SD whereas PT increased $\sim 1.4 \%$. The 5 most optimal (weighted) treatment combinations consist of the $0.1 \mathrm{~mm} \mathrm{LH}, 0.8$ SD and mostly larger ORNs (Table 10) with both levels of STS due to the relatively minor effect of the latter control variable.

\section{Discussion}

The material cost of 3D printing the model with largest MU is roughly US $\$ 20$ (MICAB model) using the Formlabs VP material in our study which would be a minor fraction of the total cost for a 3D printing laboratory situated in a developed country. However, this could be a substantially larger fraction of the total cost in a developing country and therefore would warrant a larger weighting of $\mathrm{MU}$ in contrast to the 90-10 (PT-MU) optimization scheme employed in the present work. However, the methodology presented in this study could be easily adapted for such situations that demand a more tailored weighting of PT and MU based on the specific circumstance.

The Hollow models had more than twice the support volume fraction as the Solid models primarily due to the presence of support structures in the internal geometry of these models. MU varied relatively little compared to PT within each of the 8 models, which is because PT is determined by the laser toolpaths, post-layer peel operation and miscellaneous operations all of which depend on the number of layers (model $\mathrm{Z}$ height) and layer cross-sectional area that are in turn sensitive to all 4 control variables investigated in this study. All of the most optimal treatment combinations for each of the 8 


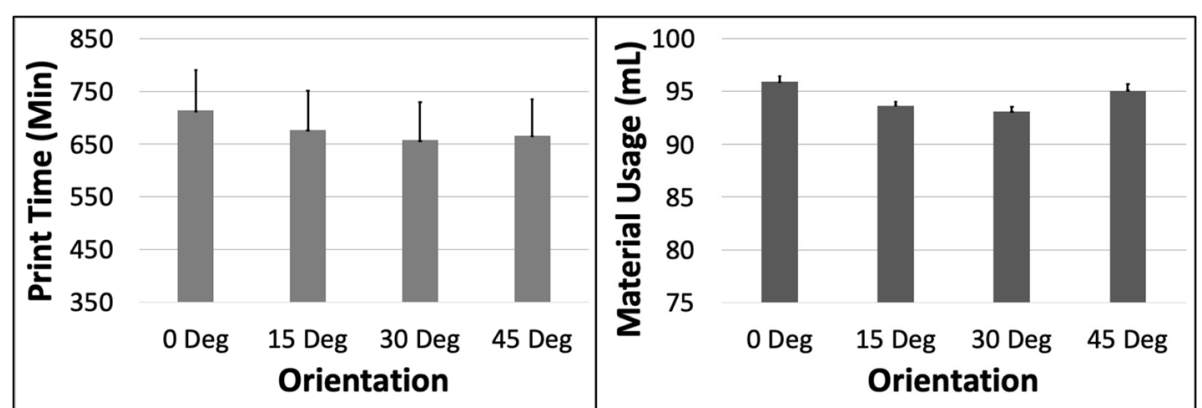

Fig. 11 The response variables Print Time (PT) and Material Usage (MU) plotted against Orientation (ORN) for the RC model. Sample size is 8 samples for each PT and MU average and the associated error bar

models consisted of the $0.1 \mathrm{~mm} \mathrm{LH}$ due to the reduced number of layers compared to the $0.05 \mathrm{~mm} \mathrm{LH}$. The reduction in PT was in the $~ 40-50 \%$ range with increased LH. In terms of ORNs both the smaller and larger levels were present in the optimal solutions since this control variable has a complex effect on PT and MU. A majority

Table 7 The 5 most weighted (W) optimal treatment combinations for the RC model

\begin{tabular}{lllllll}
\hline ORN (Deg) & LH $(\mathbf{m m})$ & SD & STS $(\mathbf{m m})$ & MU $(\mathbf{m L})$ & PT (min) & W \\
\hline 30 & 0.1 & 0.8 & 0.5 & 91.13 & 461 & -1.0000 \\
30 & 0.1 & 0.8 & 0.4 & 92.09 & 466 & -0.9510 \\
45 & 0.1 & 0.8 & 0.5 & 92.49 & 467 & -0.9348 \\
30 & 0.1 & 1 & 0.5 & 93.13 & 470 & -0.9034 \\
15 & 0.1 & 1 & 0.5 & 92.85 & 477 & -0.8854 \\
\hline
\end{tabular}

of the optimal solutions occurred at $0.8 \mathrm{SD}$ due to the negative effect of this control variable on both PT and MU, albeit not as dominant as ORN or LH. Both levels of STS were present throughout the optimal treatment combinations due to the minor effect of this variable on $\mathrm{PT}$ and MU.

The Cube model showed a reduction in both PT and MU with increasing ORN due to a reduction in Z-height with increasing ORN (Fig. 1A). The 5 most optimal treatment combinations occurred at the $0.1 \mathrm{~mm} \mathrm{LH}$ and $30 / 45$ Deg ORNs, and both these values were at the higher end of the range for both control variables. However, it is important to note that the 45 Deg ORN for the Cube would result in a flat surface on top of the support pillars and likely lead to substantial warping and reduced print quality. The optimization scheme estimates that the $0.1 \mathrm{~mm}$ LH, 30 Deg ORN, 0.8 SD and $0.5 \mathrm{~mm}$ STS would result in optimized PT and MU for the Cube model.

For the LAA model the MU initially reduced and then increased with increasing ORN. This is due to the vertical alignment of the pulmonary veins from 0 to 15 Deg that resulted in fewer support pillars followed by increasing angular alignment from 15 to 45 Deg ORN (Fig. 1B). The PT and MU both increased with SD due to the Hollow nature of the LAA model. The 5 most optimal treatment combinations consisted of both smaller (15 Deg) due to the vertical alignment of the pulmonary veins, and larger (30 and $45 \mathrm{Deg}$ ) ORNs due to the reduced Z-height. The optimization scheme estimates that the $0.1 \mathrm{~mm} \mathrm{LH}, 45 \mathrm{Deg}$ ORN, $0.8 \mathrm{SD}$ and $0.5 \mathrm{~mm}$ STS would result in optimized PT and MU for the LAA model.

For the MICAB model, the 5 most optimal treatment combinations consisted of both smaller (0 and $15 \mathrm{Deg})$

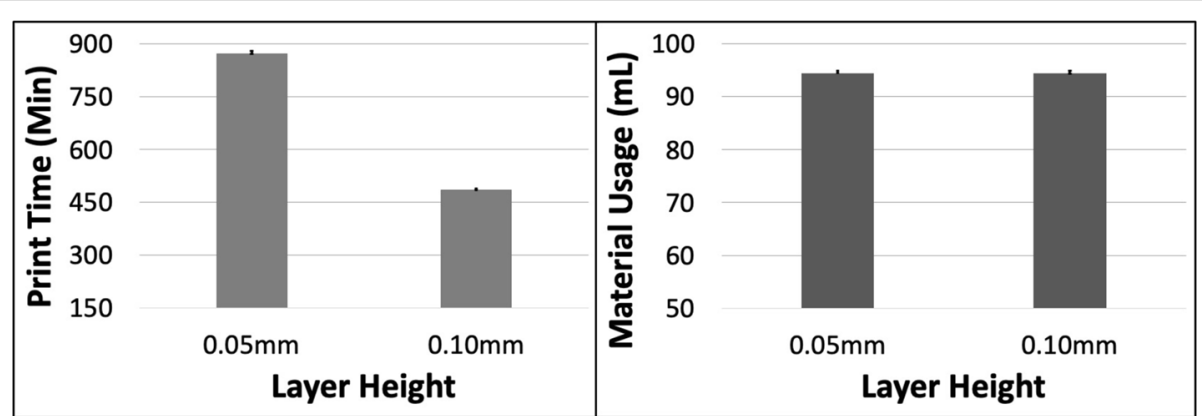

Fig. 12 The response variables Print Time (PT) and Material Usage (MU) plotted against Layer Height (LH) for the RC model. Sample size is 16 samples for each PT and MU average and the associated error bar 


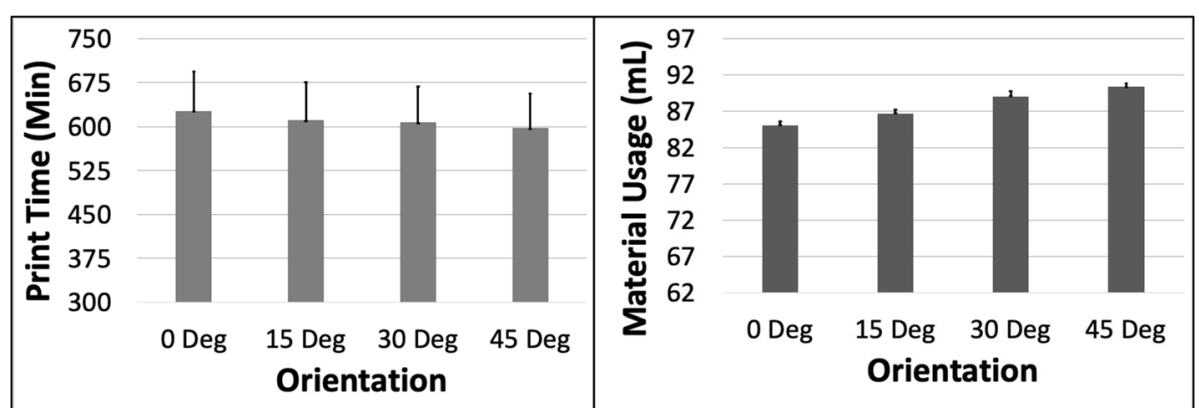

Fig. 13 The response variables Print Time (PT) and Material Usage (MU) plotted against Orientation (ORN) for the MO model. Sample size is 8 samples for each PT and MU average and the associated error bar

and larger (30 Deg) ORNs. This is due to the balance between fewer support pillars but larger Z-height in the lower ORNs and higher support pillar count but lower Z-height in the higher ORNs (Fig. 1C). The optimization scheme estimates that the $0.1 \mathrm{~mm} \mathrm{LH}, 15$ Deg ORN, 0.8 SD and $0.5 \mathrm{~mm}$ STS would result in optimized PT and MU for the MICAB model.

The PT and MU both increased with ORN for the LGG model which is due to the largest flat surface (bottom surface of the model) becoming more horizontally aligned and requiring a larger raft as well as more support pillars. Therefore, any reduction in PT due to the decrease in model Z-height with increasing ORN (Fig. 1D) was more than compensated for by an increase in support and raft volume (the opposite effect was observed in the Cube model). Four out of the 5 most optimal treatment combinations for the LGG model consisted of the 0 Deg (vertical) ORN. The optimization scheme estimates that the $0.1 \mathrm{~mm} \mathrm{LH}, 0$ Deg ORN, 0.8 $\mathrm{SD}$ and $0.5 \mathrm{~mm}$ STS would result in optimized PT and

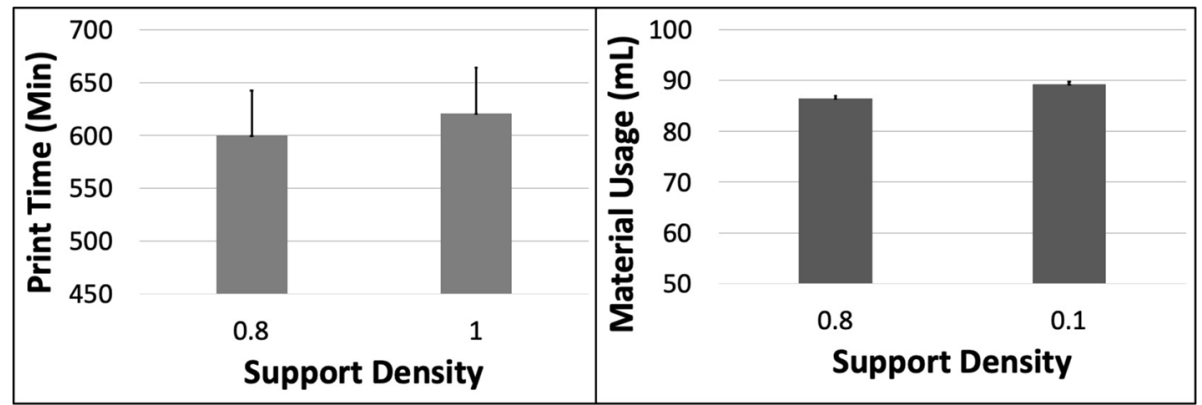

Fig. 14 The response variables Print Time (PT) and Material Usage (MU) plotted against Support Density (SD) for the MO model. Sample size is 16 samples for each PT and MU average and the associated error bar

Table 8 The 5 most weighted (W) optimal treatment combinations for the $\mathrm{MO}$ model

\begin{tabular}{lllllll}
\hline ORN (Deg) & LH $(\mathbf{m m})$ & SD & STS $(\mathbf{m m})$ & MU $(\mathbf{m L})$ & PT (min) & W \\
\hline 0 & 0.1 & 0.8 & 0.5 & 83.24 & 435 & -0.9617 \\
15 & 0.1 & 0.8 & 0.5 & 84.95 & 428 & -0.9574 \\
30 & 0.1 & 0.8 & 0.5 & 86.66 & 430 & -0.9113 \\
0 & 0.1 & 0.8 & 0.4 & 84.78 & 444 & -0.8868 \\
15 & 0.1 & 0.8 & 0.4 & 86.19 & 438 & -0.8843 \\
\hline
\end{tabular}

MU for the LGG model.

A similar balancing effect between reduced model Zheight and increased support volume with increasing ORN (Fig. 1E) was observed for the RC model as evidenced by the initially reducing PT and MU which subsequently increased with increasing ORN. Three out of the 5 most optimal treatment combinations consisted of the 30 Deg ORN, although both 0.8 and 1.0 SD levels were present. The optimization calculation estimates that the $0.1 \mathrm{~mm} \mathrm{LH}, 30$ Deg ORN, $0.8 \mathrm{SD}$ and $0.5 \mathrm{~mm}$ STS would result in optimized PT and MU for the RC model. 


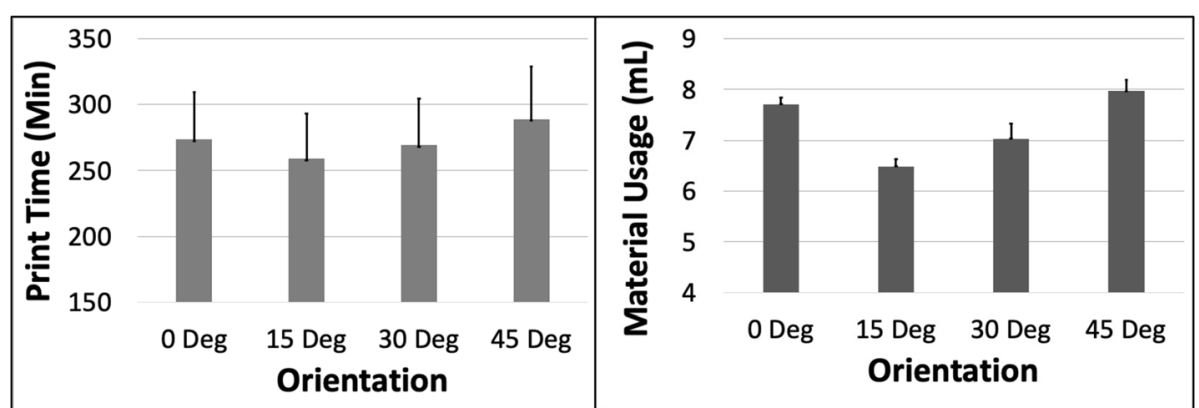

Fig. 15 The response variables Print Time (PT) and Material Usage (MU) plotted against Orientation (ORN) for the HPA model. Sample size is 8 samples for each PT and MU average and the associated error bar

The PT reduced whereas the MU increased with increasing ORN for the MO model due to the reducing Zheight and increasing support and raft volume (Fig. 1F). The slender nature of the MO model resulted in a noticeable increase in both PT and MU with increased SD. The optimization scheme estimates that the $0.1 \mathrm{~mm} \mathrm{LH}$, 0 Deg ORN, 0.8 SD and $0.5 \mathrm{~mm}$ STS would result in optimized PT and MU for the MO model. increased support and raft volume (Fig. 1G). The differences between PT and MU values at various ORNs are larger compared to other models due to the relative dominance of support/raft volume fraction $(>52 \%)$. The optimization scheme estimates that the $0.1 \mathrm{~mm} \mathrm{LH}, 15$ Deg ORN, $0.8 \mathrm{SD}$ and $0.5 \mathrm{~mm}$ STS would result in optimized PT and MU for the HPA model.

The PT decreased and MU increased with increasing

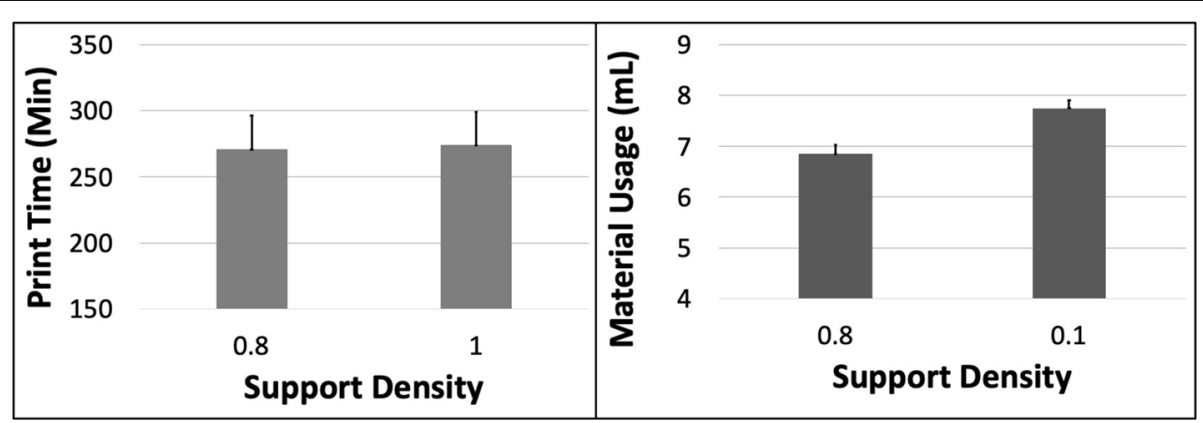

Fig. 16 The response variables Print Time (PT) and Material Usage (MU) plotted against Support Density (SD) for the HPA model. Sample size is 16 samples for each PT and MU average and the associated error bar

The PT and MU both showed "U" shaped patterns with initially decreasing and later increasing values with increasing ORN for the HPA model. This is again because of the initial benefit from reducing model Zheight which is later more than compensated for by the

Table 9 The 5 most weighted (W) optimal treatment combinations for the HPA model

\begin{tabular}{lllllll}
\hline ORN (Deg) & LH (mm) & SD & STS $(\mathbf{m m})$ & MU (mL) & PT (min) & W \\
\hline 15 & 0.1 & 0.8 & 0.5 & 6.13 & 167 & -0.9993 \\
15 & 0.1 & 0.8 & 0.4 & 6.15 & 167 & -0.9979 \\
30 & 0.1 & 0.8 & 0.4 & 6.26 & 171 & -0.9596 \\
30 & 0.1 & 0.8 & 0.5 & 6.28 & 171 & -0.9583 \\
15 & 0.1 & 1 & 0.5 & 6.87 & 171 & -0.9179 \\
\hline
\end{tabular}

ORN for the BTA model (Fig. 1H). The differences between PT and MU values at various ORNs were high similar to the HPA model due to the relative dominance of support/raft volume fraction $(>60 \%)$. The optimization scheme estimates that the $0.1 \mathrm{~mm} \mathrm{LH}, 30$ Deg ORN, $0.8 \mathrm{SD}$ and $0.4 \mathrm{~mm}$ STS would result in optimized PT and MU for the BTA model.

The study has some limitations. First, this study presents a simulation-based approach for optimizing print time and material usage for 8 different medical anatomical models (7 medical and 1 reference cube) which ignores other aspects such as print surface quality, internal support structure removal, dimensional accuracy etc. Future research must factor such additional variables into the optimization scheme. Second, it is difficult 


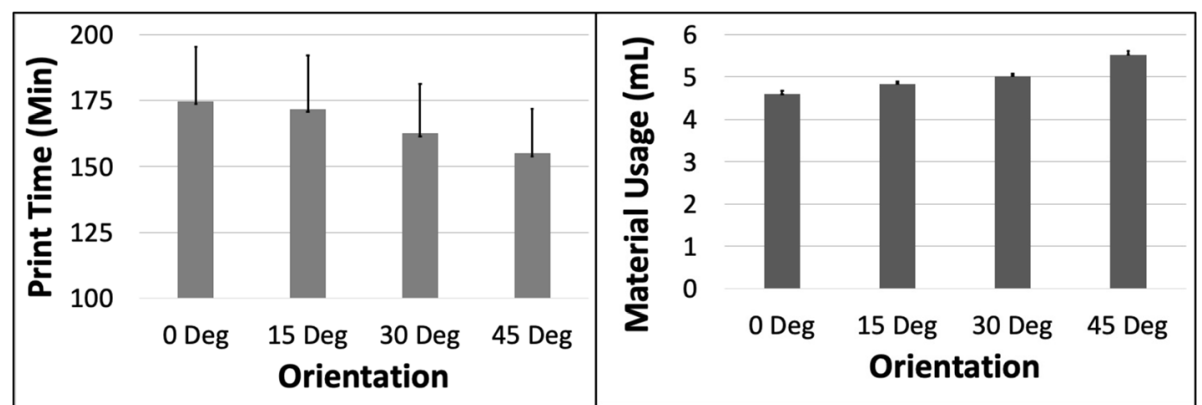

Fig. 17 The response variables Print Time (PT) and Material Usage (MU) plotted against Orientation (ORN) for the BTA model. Sample size is 8 samples for each PT and MU average and the associated error bar

to "standardize" orientations across different models; therefore, this study set the 0 Deg orientation as the vertical most orientation for each model. Third, the orientation was varied in only one degree of freedom and future studies should investigate additional degrees of freedom. Fourth, only models from single patients were used in the optimization framework and models between patients with the same conditions could vary substantially. Finally, the 90-10 weighting in the optimization scheme is subjective and in developing nations this skew would likely reduce due to availability of resources. However, the methodology presented can be adapted to concurrently optimize both print time and material usage based on the availability of resources and desired throughput for the 3D printing lab.

\section{Conclusions}

The research successfully investigated the simulated effect of orientation, layer height, support density and tip size on print time and layer height for 7 different anatomical models and 1 reference cube. Hollow models demonstrated a higher proportion of support material due to internal supports. The optimization framework identified the 5 most optimal treatment combinations for each model using a 90-10 weighting for print time and material usage. The optimal solutions all included the $0.1 \mathrm{~mm}$ layer height since the goal of the optimization scheme was to minimize both print time and material usage. Orientation had a complex effect on both print time and material usage in certain models.

Table 10 The 5 most weighted (W) optimal treatment combinations for the BTA model

\begin{tabular}{lllllll}
\hline ORN (Deg) & LH (mm) & SD & STS $(\mathbf{m m})$ & MU $(\mathbf{m L})$ & PT (min) & W \\
\hline 30 & 0.1 & 0.8 & 0.4 & 4.89 & 112 & -0.8836 \\
0 & 0.1 & 0.8 & 0.4 & 4.43 & 117 & -0.8680 \\
30 & 0.1 & 0.8 & 0.5 & 5.06 & 112 & -0.8621 \\
15 & 0.1 & 0.8 & 0.5 & 4.66 & 116 & -0.8537 \\
45 & 0.1 & 0.8 & 0.4 & 5.51 & 109 & -0.8494 \\
\hline
\end{tabular}

Support density and tip size had a relatively minor impact on both response variables. The presented optimization framework could be adapted based on the individual circumstance of each 3D printing lab and/or to potentially incorporate additional response variables of interest.

\section{Abbreviations \\ VP: vat photopolymerization; LCD: liquid crystal display; DLP: digital light projection; HU: Hounsfield units; LAA: left atrial appendage; MICAB: minimally invasive coronary artery bypass; LGG: low grade glioma; RC: renal cell carcinoma; MO: mandibular osteonecrosis; HPA: hepatic pseudoaneurysm; BTA: basilar tip aneurysm; PT: print time; MU: material usage; \\ ORN: orientation; LH: layer height; SD: support density; STS: support tip size; W: weight}

\section{Acknowledgements}

The article processing charge for this publication has been funded by an unrestricted grant from Formlabs.

\section{Authors' contributions}

PR and VC designed the study. PR performed the simulations. VC conducted the optimization analysis. PR drafted the manuscript which was revised and approved by VC. All authors read and approved the manuscript.

\section{Funding}

Not applicable.

\section{Availability of data and materials}

The raw dataset can be shared for non-commercial usage based on reasonable request.

\section{Declarations}

Ethics approval and consent to participate Not applicable.

\section{Consent for publication}

Not applicable.

\section{Competing interests}

The authors declare that they have no competing interests.

\section{Author details}

'Department of Radiology, University of Cincinnati College of Medicine, 234 Goodman St, Cincinnati, OH 45219, USA. ${ }^{2}$ Department of Industrial, Manufacturing and Systems Engineering, University of Texas at Arlington, 500 West First St, Arlington, TX 76019, USA. 
Received: 28 January 2021 Accepted: 19 July 2021

Published online: 26 August 2021

\section{References}

1. Mitsouras D, Liacouras P, Imanzadeh A, Giannopoulos AA, Cai T, Kumamaru KK, et al. Medical 3D printing for the radiologist. Radiographics. 2015;35(7): 1965-88. https://doi.org/10.1148/rg.2015140320.

2. Lichtenberger JP, Tatum PS, Gada S, Wyn M, Ho VB, Liacouras P. Using 3D printing (additive manufacturing) to produce low-cost simulation models for medical training. Mil Med. 2018;183(suppl_1):73-7. https://doi.org/10.1 093/milmed/usx142.

3. Melchels FPW, Feijen J, Grijpma DW. A review on stereolithography and its applications in biomedical engineering. Biomaterials. 2010;31 (24):6121-30. https://doi.org/10.1016/j.biomaterials.2010.04.050.

4. Ravi P, Chepelev L, Lawera N. A systematic evaluation of medical 3D printing accuracy of multi-pathological anatomical models for surgical planning manufactured in elastic and rigid material using desktop inverted vat photopolymerization. Med Phys. 2021;48(6):1-11. https://doi.org/10.1 002/mp.14850.

5. Khodaygan S, Golmohammadi AH. Multi-criteria optimization of the part build orientation (PBO) through a combined meta-modeling/NSGAII/TOPSIS method for additive manufacturing processes. Int J Interact Des Manuf. 2018;12(3):1071-85. https://doi.org/10.1007/s12008-017-0443-7.

6. Jaiswal P, Patel J, Rai R. Build orientation optimization for additive manufacturing of functionally graded material objects. Int J Adv Manuf Technol. 2018;96(1-4):223-35. https://doi.org/10.1007/s00170-018-1586-9.

7. Ravi P, Antoline S, Rybicki FJ. 3D printing of open-source respirators (including N95 respirators), surgical masks, and community mask designs to address COVID-19 shortages. In: 3D printing in medicine and its role in the COVID-19 pandemic: Springer; 2021. p. 91-106. https://doi.org/10.1007/ 978-3-030-61993-0_11.

8. Griffiths CA, Howarth J, De Almeida-Rowbotham G, et al. A design of experiments approach for the optimisation of energy and waste during the production of parts manufactured by 3D printing. J Clean Prod. 2016;139: 74-85. https://doi.org/10.1016/j.jclepro.2016.07.182.

9. Jiang J, Ma Y. Path planning strategies to optimize accuracy, quality, build time and material use in additive manufacturing: a review. Micromachines. 2020;11(7). https://doi.org/10.3390/Ml11070633.

10. Jiang J, Xu X, Stringer J. Optimization of process planning for reducing material waste in extrusion based additive manufacturing. Robot Comput Integr Manuf. 2019;59:317-25. https://doi.org/10.1016/.j.rim.2019.05.007.

11. Jiang J, Stringer J, Xu X. Support optimization for flat features via path planning in additive manufacturing. 3D print. Addit Manuf. 2019;6:171-9. https://doi.org/10.1089/3dp.2017.0124.

12. Kamio T, Hayashi K, Onda T, Takaki T, Shibahara T, Yakushiji T, et al. Utilizing a low-cost desktop 3D printer to develop a "one-stop 3D printing lab" for oral and maxillofacial surgery and dentistry fields. 3D Print Med. 2018;4:1-2. https://doi.org/10.1186/s41205-018-0028-5.

13. Rubayo DD, Phasuk K, Vickery JM, Morton D, Lin WS. Influences of build angle on the accuracy, printing time, and material consumption of additively manufactured surgical templates. J Prosthet Dent. 2020:1-6. https://doi.org/10.1016/j.prosdent.2020.09.012.

14. Salmi M, Paloheimo KS, Tuomi J, Wolff J, Mäkitie A. Accuracy of medical models made by additive manufacturing (rapid manufacturing). J CranioMaxillofacial Surg. 2013;41(7):603-9. https://doi.org/10.1016/j.jcms.2012.11.041.

15. Unkovskiy A, Bui PHB, Schille C, Geis-Gerstorfer J, Huettig F, Spintzyk S. Objects build orientation, positioning, and curing influence dimensional accuracy and flexural properties of stereolithographically printed resin. Dent Mater. 2018;34(12):e324-33. https://doi.org/10.1016/j.dental.2018.09.011.

16. Hada T, Kanazawa M, Iwaki M, Arakida T, Soeda Y, Katheng A, et al. Effect of printing direction on the accuracy of 3D-printed dentures using stereolithography technology. Materials (Basel). 2020;13(15):1-12. https://doi. org/10.3390/ma13153405.

17. Tahayeri A, Morgan MC, Fugolin AP, Bompolaki D, Athirasala A, Pfeifer CS, et al. 3D printed versus conventionally cured provisional crown and bridge dental materials. Dent Mater. 2018;34(2):192-200. https://doi.org/10.1016/j. dental.2017.10.003

\section{Publisher's Note}

Springer Nature remains neutral with regard to jurisdictional claims in published maps and institutional affiliations.

Ready to submit your research? Choose BMC and benefit from:

- fast, convenient online submission

- thorough peer review by experienced researchers in your field

- rapid publication on acceptance

- support for research data, including large and complex data types

- gold Open Access which fosters wider collaboration and increased citations

- maximum visibility for your research: over $100 \mathrm{M}$ website views per year

At BMC, research is always in progress.

Learn more biomedcentral.com/submissions 